\title{
Fixed Points of Augmented Generalized Happy Functions
}

\author{
Breeanne Baker Swart Kristen A. Beck Susan Crook \\ Christina Eubanks-Turner Helen G. Grundman \\ May Mei Laurie Zack*
}

June 4, 2022

\begin{abstract}
An augmented generalized happy function $S_{[c, b]}$ maps a positive integer to the sum of the squares of its base $b$ digits plus $c$. In this paper, we study various properties of the fixed points of $S_{[c, b]}$; count the number of fixed points of $S_{[c, b]}$, for $b \geq 2$ and $0<c<3 b-3$; and prove that, for each $b \geq 2$, there exist arbitrarily many consecutive values of $c$ for which $S_{[c, b]}$ has no fixed point.
\end{abstract}

\section{Introduction}

The concept of a happy number, defined in [5] and popularized by [3], was generalized in [2] by allowing for varying bases and exponents in the defining function. In [1, this was generalized further, altering the defining function with the addition of a constant. Specifically, for integers $c \geq 0$ and $b \geq 2$, the augmented generalized happy function, $S_{[c, b]}: \mathbb{Z}^{+} \rightarrow \mathbb{Z}^{+}$, is defined by

$$
S_{[c, b]}\left(\sum_{i=0}^{n} a_{i} b^{i}\right)=c+\sum_{i=0}^{n} a_{i}^{2},
$$

*Work on the project was partially supported by the National Science Foundation grant \#DMS 1239280. 
where $0 \leq a_{i} \leq b-1$ and $a_{n} \neq 0$. Thus, for a positive integer $a$ denoted $a_{n} \cdots a_{1} a_{0}$ in base $b$,

$$
S_{[c, b]}\left(a_{n} \ldots a_{1} a_{0}\right)=c+a_{n}^{2}+\ldots+a_{1}^{2}+a_{0}^{2} .
$$

A positive integer $a$ is a happy number if for some $k \in \mathbb{Z}^{+}, S_{[0,10]}^{k}(a)=1$. Although 1 is the sole fixed point of $S_{[0,10]}$, as shown in [2], for $b \neq 10, S_{[0, b]}$ may have additional fixed points. Similarly, as shown in [1], when $c>0$ (and $b \geq 2), S_{[c, b]}$ may have multiple fixed points.

In this work, we study the fixed points of the functions $S_{[c, b]}$. First, in Section 2, we prove some preliminary results providing properties of the fixed points and consecutive fixed points of an arbitrary, but fixed, $S_{[c, b]}$. Then, in Section 3, we discuss the exact number of fixed points of $S_{[c, b]}$, in terms of $c$ and $b$. Finally, in Section 4 , we let $c \geq 0$ vary and prove that for each $b \geq 2$, there are arbitrarily long sequences of consecutive values of $c$ for which $S_{[c, b]}$ has no fixed point.

\section{Fixed Point Characteristics}

Here we discuss a variety of results concerning relationships between the fixed

points of a single $S_{[c, b]}$, where $c \geq 0$ and $b \geq 2$ are arbitrary integers. The first theorem concerns consecutive fixed points of $S_{[c, b]}$.

Theorem 2.1. Fix $c \geq 0$ and $b \geq 2$.

1. If $a \in \mathbb{Z}^{+}$is a multiple of $b$, then $a$ is a fixed point of $S_{[c, b]}$ if and only if $a+1$ is a fixed point of $S_{[c, b]}$.

2. Every consecutive pair of fixed points of $S_{[c, b]}$ has a multiple of $b$ as its first member.

3. There is no consecutive triplet of fixed points of $S_{[c, b]}$.

Proof. We begin with the proof of Part 1. Since $a$ is a multiple of $b$, we have $S_{[c, b]}(a+1)=S_{[c, b]}(a)+1^{2}=S_{[c, b]}(a)+1$. Thus $S_{[c, b]}(a)=a$ if and only if $S_{[c, b]}(a+1)=a+1$.

For Part 2, assume that $a$ and $a+1$ are both fixed points of $S_{[c, b]}$ and using standard notation for base $b$, let

$$
a=\sum_{i=0}^{n} a_{i} b^{i}
$$


First, assume that $a_{0} \neq b-1$. Then

$$
\begin{aligned}
a & =S_{[c, b]}(a)=c+\sum_{i=1}^{n} a_{i}^{2}+a_{0}^{2}, \quad \text { and so } \\
a+1 & =S_{[c, b]}(a+1)=c+\sum_{i=1}^{n} a_{i}^{2}+\left(a_{0}+1\right)^{2} \\
& =a+2 a_{0}+1 .
\end{aligned}
$$

Thus, $2 a_{0}=0$, implying that $a_{0}=0$. Therefore $a$ is a multiple of $b$, as desired.

Next assume, for a contradiction, that $a_{0}=b-1$. Let $j \in \mathbb{Z}^{+}$be minimal such that $a_{j} \neq b-1$. (If every digit of $a$ is equal to $b-1$, then $a+1=b^{k}$ for some natural number $k$ and $S_{[c, b]}(a+1)=1$, so $a+1$ is not a fixed point.) Then

$$
a=S_{[c, b]}(a)=c+\sum_{i=j}^{n} a_{i}^{2}+j(b-1)^{2}
$$

and since

$$
\begin{aligned}
& a+1=\sum_{i=j+1}^{n} b^{i} a_{i}+\left(a_{j}+1\right) b^{j}, \quad \text { we have } \\
& a+1=S_{[c, b]}(a+1)=c+\sum_{i=j+1}^{n} a_{i}^{2}+\left(a_{j}+1\right)^{2} .
\end{aligned}
$$

Combining equations (21) and (3) yields

$$
a_{j}^{2}+j(b-1)^{2}+1=\left(a_{j}+1\right)^{2} .
$$

Thus, $j(b-1)^{2}=2 a_{j}$. Since $a_{j}<b-1, j(b-1)<2$ and so $j=b-1=1$. But then $2 a_{j}=1$, which is a contradiction.

Finally, Part 3 is immediate from Part 2.

Lemma 2.2 provides another pairing of fixed points of $S_{[c, b]}$.

Lemma 2.2. Fix $c \geq 0, b \geq 2$, and $a \in \mathbb{Z}^{+}$where

$$
a=\sum_{i=0}^{n} a_{i} b^{i}
$$


in standard base $b$ notation with $a_{1} \neq 0$. Let

$$
\tilde{a}=\sum_{i=2}^{n} a_{i} b^{i}+\left(b-a_{1}\right) b+a_{0} .
$$

Then a is a fixed point of $S_{[c, b]}$ if and only if $\tilde{a}$ is a fixed point of $S_{[c, b]}$.

Proof. Assume that $a$ and $\tilde{a}$ are as above, and that $a$ is a fixed point of $S_{[c, b]}$. Then

$$
\begin{aligned}
S_{[c, b]}(\tilde{a}) & =S_{[c, b]}\left(\sum_{i=2}^{n} a_{i} b^{i}+\left(b-a_{1}\right) b+a_{0}\right)=c+\sum_{i=2}^{n} a_{i}^{2}+\left(b-a_{1}\right)^{2}+a_{0}^{2} \\
& =c+\sum_{i=0}^{n} a_{i}^{2}+b^{2}-2 a_{1} b=S_{[c, b]}(a)+b^{2}-2 a_{1} b=a+b^{2}-2 a_{1} b \\
& =\sum_{i=0}^{n} a_{i} b^{i}+\left(b-2 a_{1}\right) b=\sum_{i=2}^{n} a_{i} b^{i}+\left(b-a_{1}\right) b+a_{0}=\tilde{a} .
\end{aligned}
$$

Therefore $\tilde{a}$ is also a fixed point of $S_{[c, b]}$. The converse is immediate by symmetry.

Finally, we consider the parity of $c$ that is required for $S_{[c, b]}$ to have a fixed point.

Lemma 2.3. Fix $c \geq 0$ and $b \geq 2$, and let $a=\sum_{i=0}^{n} a_{i} b^{i}$ be a fixed point of $S_{[c, b]}$, in the usual base $b$ notation.

1. If $b$ is odd, then $c$ is even.

2. If $b$ is even, then $c \equiv \sum_{i=1}^{n} a_{i}(\bmod 2)$.

Proof. For $b$ odd, by [1, Lemma 2.3], $S_{[c, b]}(a) \equiv c+a(\bmod 2)$, which implies that $c$ is even. For $b$ even, since $a$ is a fixed point of $S_{[c, b]}$,

$$
a_{0} \equiv a=S_{[c, b]}(a)=c+\sum_{i=0}^{n} a_{i}^{2} \equiv c+\sum_{i=0}^{n} a_{i} \quad(\bmod 2) .
$$

Subtracting $a_{0}$ from both sides of the congruence yields the result. 


\section{Counting the Number of Fixed Points}

In this section, we consider the number of fixed points of the function $S_{[c, b]}$ for fixed $c \geq 0$ and $b \geq 2$. In Corollary 3.5, we provide a formula for the number of fixed points of $S_{[c, b]}$ for all values of $b$ and a range of values of $c$, depending on $b$.

We begin by determining the number of fixed points of $S_{[c, b]}$ of the form $u b^{n}$, where $0<u<b$ and $n \geq 0$. To fix notation, for $c \geq 0, b \geq 2$, and $n \geq 0$, let

$$
\mathcal{F}_{[c, b]}^{(n)}=\left\{a=u b^{n} \mid 0<u<b \text { and } S_{[c, b]}(a)=a\right\} .
$$

In the following three lemmas, we provide conditions under which $\mathcal{F}_{[c, b]}^{(n)}$ assumes specified values.

Lemma 3.1. Fix $b \geq 2$. For $c>0, \mathcal{F}_{[c, b]}^{(0)}$ is empty, while $\mathcal{F}_{[0, b]}^{(0)}=\{1\}$.

Proof. Let $0<a<b$. Then $a=S_{[c, b]}(a)$ implies that $c=a-a^{2} \leq 0$. Hence, if $c=0$, we have $a=1$, and if $c>0$, we have a contradiction.

Lemma 3.2. Fix $c \geq 0$ and $b \geq 2$. The cardinality of $\mathcal{F}_{[c, b]}^{(1)}$ is

$$
\left|\mathcal{F}_{[c, b]}^{(1)}\right|= \begin{cases}2 & \text { if } \alpha^{2}-\alpha b+c=0 \text { for some integer } 1 \leq \alpha<\frac{1}{2} b \\ 1 & \text { if } b^{2}=4 c, \text { and } \\ 0 & \text { otherwise. }\end{cases}
$$

Proof. From the definition of $\mathcal{F}_{[c, b]}^{(1)}$, given $a=u b$ with $0<u<b$, we have $a \in \mathcal{F}_{[c, b]}^{(1)}$ if and only if $S_{[c, b]}(a)=a$ or, equivalently, $c+u^{2}=u b$. Thus, $\left|\mathcal{F}_{[c, b]}^{(1)}\right| \neq 0$ if and only if there exists some integer $u, 0<u<b$, such that

$$
u^{2}-u b+c=0
$$

Since this is a quadratic equation, there are at most two such values of $u$, and at most one if $b^{2}=4 c$.

By Lemma 2.2, $a=u b$ is a fixed point of $S_{[c, b]}$ if and only if $\tilde{a}=(b-u) b$ is a fixed point of $S_{[c, b]}$. Hence $a=u b \in \mathcal{F}_{[c, b]}^{(1)}$ if and only if $(b-u) b \in \mathcal{F}_{[c, b]}^{(1)}$. Thus, $\left|\mathcal{F}_{[c, b]}^{(1)}\right|=2$ if and only if there are two integer solutions to equation (44) with $0<u<b$, in which case one of the solutions will satisfy $1 \leq u<\frac{1}{2} b$. The lemma follows. 
Lemma 3.3. For $c \geq 0, b \geq 2$, and $n \geq 2$, the cardinality of $\mathcal{F}_{[c, b]}^{(n)}$ is

$$
\left|\mathcal{F}_{[c, b]}^{(n)}\right|= \begin{cases}1 & \text { if } b^{2 n}-4 c \text { is a nonzero perfect square, and } \\ 0 & \text { otherwise. }\end{cases}
$$

Proof. Fix $n \geq 2$, and suppose that $a=u b^{n}$ is a fixed point of $S_{[c, b]}$ for some $0<u<b$. Then $u b^{n}=a=S_{[c, b]}(a)=c+u^{2}$, which implies that

$$
u=\frac{b^{n} \pm \sqrt{b^{2 n}-4 c}}{2} .
$$

Since $u \in \mathbb{Z}^{+}, b^{2 n}-4 c$ is a perfect square, and since $u<b$ and $n \geq 2$, $b^{2 n}-4 c$ is nonzero. Conversely, if $b^{2 n}-4 c$ is a nonzero perfect square, then $\frac{b^{n}+\sqrt{b^{2 n}-4 c}}{2}>b$ and so is not a candidate for $u$, while, letting $u=\frac{b^{n}-\sqrt{b^{2 n}-4 c}}{2}$, it is easily verified that $a=u b^{n} \in \mathcal{F}_{[c, b]}^{(n)}$.

The following theorem and its proof were inspired by the work of Hargreaves and Siksek [4] on the number of fixed points of (unaugmented) generalized happy functions. As is standard, we let $r_{2}(n)$ denote the number of representations of $n \in \mathbb{Z}^{+}$as the sum of two squares; that is,

$$
r_{2}(n)=\left|\left\{(x, y) \in \mathbb{Z}^{2} \mid x^{2}+y^{2}=n\right\}\right| .
$$

Theorem 3.4. For $c>0$ and $b \geq 2$, the number of two-digit fixed points of $S_{[c, b]}$ is given by

$$
\begin{cases}\frac{1}{2} r_{2}\left(b^{2}-4 c+1\right)+\left|\mathcal{F}_{[c, b]}^{(1)}\right| & \text { if } b \text { is odd, and } \\ \frac{1}{4} r_{2}\left(b^{2}-4 c+1\right)+\left|\mathcal{F}_{[c, b]}^{(1)}\right| & \text { if } b \text { is even } .\end{cases}
$$

Proof. Note that $a=u b+v$ is a fixed point of $S_{[c, b]}$, with $0<u<b$ and $0 \leq v<b$ if and only if $u b+v=S_{[c, b]}(u b+v)=c+u^{2}+v^{2}$. Define

$$
U=\left\{(u, v) \in \mathbb{Z}^{2} \mid 0<u, v<b \text { and } u b+v=c+u^{2}+v^{2}\right\} .
$$

By the correspondence $(u, v) \leftrightarrow u b+v,|U|$ is equal to the number of twodigit fixed points of $S_{[c, b]}$ that are not multiples of $b$. Hence, the number of two-digit fixed points of $S_{[c, b]}$ is equal to $|U|+\left|\mathcal{F}_{[c, b]}^{(1)}\right|$. 
Set

$$
X=\left\{(x, y) \in \mathbb{Z}^{2} \mid y \geq 1 \text { odd, and } x^{2}+y^{2}=b^{2}-4 c+1\right\} .
$$

To see that $|U|=|X|$, consider the functions $\phi: U \rightarrow X$ and $\psi: X \rightarrow U$ defined by

$$
\phi(u, v)=(2 u-b, 2 v-1) \quad \text { and } \quad \psi(x, y)=\left(\frac{x+b}{2}, \frac{y+1}{2}\right) .
$$

A straightforward calculation, and noting that $2 v-1>0$ and odd, shows that the image of $\phi$ is contained in $X$. Let $(x, y) \in X$, to see that $\psi(x, y) \in U$, first note that $y$ is odd and $x \equiv b(\bmod 2)$, and so $\psi(x, y) \in \mathbb{Z}^{2}$. Next, since $x^{2}<x^{2}+y^{2}=b^{2}-4 c+1<b^{2}$, we have $-b<x<b$, implying that $0<x+b<2 b$, and thus $0<(x+b) / 2<b$, as desired. Similarly, $1 \leq y<b$, so $1 \leq(y+1) / 2<b$. Finally, a direct calculation verifies that the needed equation is satisfied.

Since, as is easily checked, $\phi$ and $\psi$ are inverses, it follows that $|U|=|X|$.

Now, note that $X$ is a subset of

$$
Z=\left\{(x, y) \in \mathbb{Z}^{2} \mid x^{2}+y^{2}=b^{2}-4 c+1\right\},
$$

and recall that, by equation ([6),,$|Z|=r_{2}\left(b^{2}-4 c+1\right)$.

If $b$ is odd and $(x, y) \in Z$, then $b^{2}-4 c+1 \equiv 2(\bmod 4)$, and so $y$ must be odd. Thus $\varphi_{\text {odd }}: Z \rightarrow X$ defined by $(x, y) \mapsto(x,|y|)$ is a 2-to-1 surjective function. Hence $|X|=\frac{1}{2}|Z|=\frac{1}{2} r_{2}\left(b^{2}-4 c+1\right)$.

If $b$ is even and $(x, y) \in Z$, then $b^{2}-4 c+1$ is odd, and so exactly one of $x$ and $y$ is odd. Thus $\varphi_{\text {even }}: Z \rightarrow X$ defined by

$$
(x, y) \mapsto \begin{cases}(x,|y|) & \text { if } y \text { is odd } \\ (y,|x|) & \text { if } x \text { is odd }\end{cases}
$$

is a 4 -to- 1 surjective function. Hence $|X|=\frac{1}{4}|Z|=\frac{1}{4} r_{2}\left(b^{2}-4 c+1\right)$.

Recalling that the number of two-digit fixed points of $S_{[c, b]}$ is $|U|+\left|\mathcal{F}_{[c, b]}^{(1)}\right|=$ $|X|+\left|\mathcal{F}_{[c, b]}^{(1)}\right|$, the result follows.

Corollary 3.5. For $b \geq 2$ and $0<c<3 b-3$, the number of fixed points of $S_{[c, b]}$ is exactly

$$
\begin{cases}\frac{1}{2} r_{2}\left(b^{2}-4 c+1\right)+\left|\mathcal{F}_{[c, b]}^{(1)}\right| & \text { if } b \text { is odd, and } \\ \frac{1}{4} r_{2}\left(b^{2}-4 c+1\right)+\left|\mathcal{F}_{[c, b]}^{(1)}\right| & \text { if } b \text { is even } .\end{cases}
$$


Proof. By [1, Lemma 2.2], since $c<3 b-3$, for each $a>b^{2}, S(a)<a$ and, therefore, $a$ is not a fixed point. Hence each fixed point of $S_{[c, b]}$ has at most two digits. The corollary now follows directly from Lemma 3.1 and Theorem 3.4 .

\section{Fixed Point Deserts}

In this section, we fix the base $b \geq 2$ and consider consecutive values of $c$ for which $S_{[c, b]}$ has no fixed points. Note that for a fixed $b$, if $a=\sum_{i=0}^{n} a_{i} b^{i}$ is a fixed point of $S_{[c, b]}$, with $0 \leq a_{i}<b$, for each $i$, then, solving for $c$, we have

$$
c=\sum_{i=0}^{n} a_{i}\left(b^{i}-a_{i}\right) .
$$

Definition 1 . For $b \geq 2$ and $k \in \mathbb{Z}^{+}$, an $k$-desert base $b$ is a set of $k$ consecutive non-negative integers $c$ for each of which $S_{[c, b]}$ has no fixed points. A desert base $b$ is an $k$-desert base $b$ for some $k \geq 1$.

For example, for $28 \leq c \leq 35, S_{[c, 10]}$ has no fixed points and, therefore, there is an 8-desert base 10 starting at $c=28$.

We begin by determining bounds on the values of $c$ such that $S_{[c, b]}$ has a fixed point of a given number of digits. For $b \geq 2$ and $n \geq 2$ define

$$
\begin{aligned}
& m_{b, n}=b^{n}-b^{2}+3 b-3, \text { and } \\
& M_{b, n}=b^{n+1}-b^{2}-(n-1)(b-1)^{2}+(b-\lfloor b / 2\rfloor)\lfloor b / 2\rfloor .
\end{aligned}
$$

Theorem 4.1. Let $b \geq 2$ and $n \geq 2$. If $S_{[c, b]}$ has a $n+1$-digit fixed point, then $m_{b, n} \leq c \leq M_{b, n}$. Further, these bounds are sharp.

Proof. Let $b \geq 2$ and $n \geq 2$ be fixed. By equation (7), each fixed point $a$ of $S_{[c, b]}$ determines the value of $c$. Treating the $a_{i}$ in equation (7) as independent variables taking on integer values between 0 and $b-1$, inclusive, we find the minimal possible value of $c$ by minimizing each term. Observe that $a_{0}\left(b^{0}-a_{0}\right)$ is minimal when $a_{0}=b-1$; for $0<i<n, a_{i}\left(b^{i}-a_{i}\right)$ is minimal when $a_{i}=0$; and, since $a_{n} \neq 0, a_{n}\left(b^{n}-a_{n}\right)$ is minimal when $a_{n}=1$. Hence the minimal value of $c$ is determined by

$$
a=\sum_{i=0}^{n} a_{i}^{\prime} b^{i}, \text { where } a_{i}^{\prime}= \begin{cases}1, & \text { for } i=n \\ 0, & \text { for } 1 \leq i \leq n-1 \\ b-1, & \text { for } i=0\end{cases}
$$


and so, the minimal value of $c$ is

$$
c=(b-1)\left(b^{0}-(b-1)\right)+1 \cdot\left(b^{n}-1\right)=b^{n}-b^{2}+3 b-3=m_{b, n} .
$$

Similarly, maximizing the terms of equation (7), we find that $a_{0}\left(b^{0}-a_{0}\right)$ is maximal when $a_{0}=0 ; a_{1}\left(b^{1}-a_{1}\right)$ is maximal when $a_{1}=\lfloor b / 2\rfloor$; and for $1<i \leq n, a_{i}\left(b^{i}-a_{i}\right)$ is maximal when $a_{i}=b-1$. Hence, the maximal value of $c$ is determined by

$$
a=\sum_{i=0}^{n} a_{i}^{\prime \prime} b^{i}, \text { where } a_{i}^{\prime \prime}= \begin{cases}b-1, & \text { for } 2 \leq i \leq n \\ \left\lfloor\frac{b}{2}\right\rfloor, & \text { for } i=1 \\ 0, & \text { for } i=0\end{cases}
$$

and, therefore, the maximal value of $c$ is

$$
\begin{aligned}
c & =\lfloor b / 2\rfloor\left(b^{1}-\lfloor b / 2\rfloor\right)+\sum_{i=2}^{n}\left(b^{i}-(b-1)\right)(b-1) \\
& =b^{n+1}-b^{2}-(n-1)(b-1)^{2}+(b-\lfloor b / 2\rfloor)\lfloor b / 2\rfloor=M_{b, n} .
\end{aligned}
$$

The following lemma is used to prove Theorem 4.3, which states that for each $b \geq 2$ there exist arbitrarily long deserts base $b$.

Lemma 4.2. Let $b \geq 2$ and $n \geq 2$. Then, between the numbers $M_{b, n}$ and $m_{b, n+1}$, there exists an $k$-desert base $b$, where

$$
k=m_{b, n+1}-M_{b, n}-1>(n-5 / 4)(b-1)^{2} .
$$

Proof. Let $b \geq 2$ and $n \geq 2$ be fixed. Note that

$$
\begin{aligned}
m_{b, n+1}-M_{b, n}-1= & \left(b^{n+1}-b^{2}+3 b-3\right)- \\
& \quad\left(b^{n+1}-b^{2}-(n-1)(b-1)^{2}+(b-\lfloor b / 2\rfloor)\lfloor b / 2\rfloor\right)-1 \\
\geq & 3 b-3+(n-1)(b-1)^{2}-b^{2} / 4-1 \\
= & (n-1)(b-1)^{2}-b^{2} / 4+b / 2-1 / 4+5 b / 2-15 / 4 \\
> & (n-1)(b-1)^{2}-(b-1)^{2} / 4 \\
= & (n-5 / 4)(b-1)^{2},
\end{aligned}
$$

since $b \geq 2$. Thus,

$$
m_{b, n+1}>M_{b, n}+1 \text {. }
$$


Recall that $M_{b, n}$ is an upper bound on values of $c$ such that $S_{[c, b]}$ has a $(n+1)$ digit fixed point. Since $M_{b, x}$ increases as $x$ increases, $M_{b, n}$ is an upper bound on values of $c$ such that $S_{[c, b]}$ has a fixed point with less than or equal to $(n+1)$-digits. Similarly, $m_{b, n+1}$ is a lower bound on values of $c$ such that $S_{[c, b]}$ has a fixed point with greater than or equal to $(n+2)$-digits.

Thus, by equation (8), there is no value of $c$ between $M_{b, n}$ and $m_{b, n+1}$ such that $S_{[c, b]}$ has a fixed point of any size. Hence there exists an $k$-desert between these two numbers, where $k=m_{b, n+1}-M_{b, n}-1$.

Theorem 4.3. For each $b \geq 2$ and $k \in \mathbb{Z}^{+}$, there exists an $k$-desert base $b$.

Proof. Fix $b \geq 2$ and $k \in \mathbb{Z}^{+}$. Since $(n-5 / 4)(b-1)^{2}$ is an increasing linear function of $n$, there exists some $n \geq 2$ such that $(n-5 / 4)(b-1)^{2} \geq k$. It follows from Lemma 4.2 that there exists an $k$-desert base $b$.

\section{References}

[1] B. Baker Swart, K. Beck, S. Crook, C. Eubanks-Turner, H. Grundman, M. Mei, and L. Zack, Augmented gemeralized happy functions, Rocky Mountain Journal of Mathematics, to appear.

[2] H. G. Grundman and E. A. Teeple, Generalized happy numbers, Fibonacci Quart. 39 (2001), no. 5, 462-466.

[3] Richard K. Guy, Unsolved problems in number theory, third ed., Problem Books in Mathematics, Springer-Verlag, New York, 2004.

[4] K. Hargreaves and S. Siksek, Cycles and fixed points of happy functions, Journal of Combinatorics and Number Theory, 2 (2010), no. 3, 65-77.

[5] Ross Honsberger, Ingenuity in Mathematics, New Mathematical Library, 23, Random House, Inc., New York, 1970. 